\title{
Diabetes insipidus secondary to nivolumab-induced neurohypophysitis and pituitary metastasis
}

\author{
Michele Fosci(1) 1, Francesca Pigliaru1, Antonio Stefano Salcuni¹, Massimo Ghiani², \\ Maria Valeria Cherchi³, Maria Antonietta Calia ${ }^{4}$, Andrea Loviselli ${ }^{1}$ and \\ Fernanda Velluzzi ${ }^{1}$
}

1Department of Medical Sciences, University of Cagliari, Azienda Universitaria-Ospedaliera of Cagliari, Cagliari, Sardegna, Italy, 2Department of Medical Oncology, Azienda Ospedaliera Brotzu, Cagliari, Sardegna, Italy, 3 Department of Radiology, University of Cagliari, Azienda Universitaria-Ospedaliera of Cagliari, Cagliari, Sardegna, Italy, and 4ATS Sardegna, ASL 8, Servizio di Endocrinologia Cagliari, Sardegna, Italy
Correspondence should be addressed to M Fosci

Email

michele.fosci92@gmail.com

\section{Summary}

A 62-year-old patient with metastatic hypopharyngeal carcinoma underwent treatment with nivolumab, following which he developed symptoms suggestive of diabetes insipidus. Nivolumab was stopped and therapy with methylprednisolone was started. During corticosteroid therapy, the patient presented himself in poor health condition with fungal infection and glycemic decompensation. Methylprednisolone dose was tapered off, leading to the resolution of mycosis and the restoration of glycemic compensation, nevertheless polyuria and polydipsia persisted. Increase in urine osmolarity after desmopressin administration was made diagnosing central diabetes insipidus as a possibility. The neuroradiological data by pituitary MRI scan with gadolinium was compatible with coexistence of metastatic localization and infundibuloneurohypophysitis secondary to therapy with nivolumab. To define the exact etiology of the pituitary pathology, histological confirmation would have been necessary; however, unfortunately, it was not possible. In the absence of histological confirmation, we believe it is likely that both pathologies coexisted.

\section{Learning points:}

- A remarkable risk of endocrine immune-related adverse events (irAEs) during therapy with checkpoint inhibitors exsists.

- In order to ensure maximum efficiency in the recognition and treatment of endocrine iRAes related to immune checkpoint inhibitors, multidisciplinary management of oncological patients is critical.

- The pituitary syndrome in oncological patients who underwent immunotherapy represents a challenge in the differential diagnosis between pituitary metastasis and drug-induced hypophysitis.

- This is the first case, described in the literature of diabetes insipidus in a patient suffering from nivolumab-induced infundibulo-neurohypophysitis and anterohypophyseal metastasis.

\section{Background}

The introduction of immune checkpoint inhibitors into oncological clinical practice has revolutionized the treatment of various neoplastic diseases. These drugs awaken the anti-tumor immune response by interrupting the inhibitory signal pathways and promote immunemediated elimination of cancer cells. The targets of these therapies include CTLA-4 (cytotoxic T-lymphocyte associated protein-4), PD1 (programmed cell death 
protein-1) and PD-L1 (programmed death ligand-1). This mechanism is also the basis of immune-related adverse events (iRAes), due to the inhibition of immunecheckpoints that reinforce normal physiological barriers against autoimmunity (1). The main adverse events affect the skin, the gastrointestinal, musculoskeletal and endocrine systems. The main endocrinological manifestations are represented by thyroid dysfunction and hypophysitis. The involvement of the posterior pituitary, and consequent diabetes insipidus is exceptional, but not impossible $(2,3,4)$. Pituitary metastasis is a rare complication of advanced malignancy; however, almost every cancer type can metastasize to the pituitary fossa. Diabetes insipidus is common among patients with pituitary metastasis (5).

\section{Case presentation}

We present the case of a 62 -year-old patient with metastatic hypopharyngeal carcinoma undergoing treatment with Nivolumab (anti- PD1 MAB), following which he developed central diabetes insipidus. In 2002, the patient, with a history of heavy smoking and in the absence of other comorbidities, received the diagnosis of hypopharyngeal carcinoma and was treated with chemotherapy (platinum and fluorine) and surgery. In 2016, relapse on the lingual body developed, and due to the inoperability of the lesion, chemotherapy with cisplatin, 5-fluorouracil and erbitux was provided. In 2017, PET showed lung disease with massive metastatic adenopathies in almost all mediastinal lymph node stations. It was decided to carry out a re-challenge with platinum with evidence in January 2019 of reduction of lung and lymph node lesions. The CT of May 2019 showed a progression of pathology upon which the oncologist decided to start therapy with vivolumab (240 mg biweekly). After about 5 weeks of treatment (before the fourth cycle of therapy), the patient reported sudden onset of polyuria and polydipsia. The endocrine tests he underwent (Table 1) suggested the presence of hypophysitis as a result of mild hyperprolactinemia $(19.8 \mathrm{ng} / \mathrm{mL})$ and hypogonadotropic hypogonadism, in association with symptoms suggestive of diabetes insipidus. Moreover, the routine laboratory investigations (Table 2) revealed dehydration (hypercalcemia, hypernatremia, hyperkalemia) and impaired fasting glucose (blood glucose: $116 \mathrm{mg} / \mathrm{dL}$ ); glycated hemoglobin (HbA1c) and oral glucose tolerance test (OGTT) were not sought. Considered the onset of a possible hypophysitis, the oncologist stopped nivolumab temporarily and started therapy with methylprednisolone $64 \mathrm{mg} /$ day and
Table 1 Laboratory values after 5 weeks of oncological treatment.

\begin{tabular}{l}
\hline Hormone \\
\hline TSH, $\mu \mathrm{UI} / \mathrm{mL}$ \\
FT3, pg/mL \\
FT4, pg/mL \\
Testosterone, $\mathrm{ng} / \mathrm{mL}$ \\
$\mathrm{LH}, \mathrm{mUI} / \mathrm{mL}$ \\
$\mathrm{FSH}, \mathrm{mUI} / \mathrm{mL}$ \\
Prolactin, $\mathrm{ng} / \mathrm{mL}$ \\
ACTH, $\mu \mathrm{gL}$ \\
Cortisol, pg/mL \\
\hline
\end{tabular}

\begin{tabular}{c}
\hline Result \\
\hline 1.00 \\
3.12 \\
1.25 \\
0.5 \\
3.0 \\
2.7 \\
19.8 \\
19.2 \\
20.4 \\
\hline
\end{tabular}

\begin{tabular}{c} 
Reference values \\
\hline $0.55-4.78$ \\
$2-4.2$ \\
$0.8-1.7$ \\
$1.39-9.13$ \\
$1.4-18.1$ \\
$4.5-9.3$ \\
$2.1-17.7$ \\
$5.27-22.45$ \\
$10-60$
\end{tabular}

requested endocrinological advice. After about 10 days of corticosteroid therapy, the patient was hospitalized in the endocrinology department. He presented in a poor health condition, in a state of dehydration and with a severe fungal infection of the oral cavity. Asthenia persisted,

Table 2 Blood count and biochemistry test after 5 weeks of oncological treatment.

\begin{tabular}{|c|c|c|}
\hline $\begin{array}{l}\text { Blood count and } \\
\text { biochemistry test }\end{array}$ & Result & Reference values \\
\hline $\mathrm{WBC}, \times 10^{3} / \mu \mathrm{L}$ & 11.25 & $4.3-10.5$ \\
\hline $\mathrm{RBC}, \times 10^{6} / \mu \mathrm{L}$ & 5.50 & $4.2-5.8$ \\
\hline Hemoglobin, g/dL & 16.1 & $12-17.5$ \\
\hline Hematocrit, $\%$ & 50.5 & $37-52$ \\
\hline MCV, flL & 91.8 & $80-99$ \\
\hline $\mathrm{MCH}, \mathrm{pg}$ & 29.3 & $27-32$ \\
\hline $\mathrm{MCHC}, \mathrm{g} / \mathrm{dL}$ & 31.9 & $33-37$ \\
\hline RDW, \% & 15.1 & $11.5-14.5$ \\
\hline Platelets, $\times 10^{3} / \mu \mathrm{L}$ & 205 & $130-400$ \\
\hline Neutrophils, $\times 10^{3} / \mu \mathrm{L}$ & 8.30 & $1.9-8$ \\
\hline Lymphocytes, $\times 10^{3} / \mu \mathrm{L}$ & 1.86 & $0.9-5.2$ \\
\hline Monocytes, $\times 10^{3} / \mu \mathrm{L}$ & 0.88 & $0.2-1$ \\
\hline Eosinophils, $\times 10^{3} / \mu \mathrm{L}$ & 0.15 & $0-0.8$ \\
\hline Basophils, $\times 10^{3} / \mu \mathrm{L}$ & 0.07 & $0-0.2$ \\
\hline Glucose, mg/dL & 116 & $60-100$ \\
\hline $\mathrm{BUN}, \mathrm{mg} / \mathrm{dL}$ & 9 & $5-25$ \\
\hline Creatinine, $\mathrm{mg} / \mathrm{dL}$ & 0.8 & $0.6-1.2$ \\
\hline Sodium, mEq/L & 150 & $136-146$ \\
\hline Potassium, mEq/L & 5.3 & $3.5-5.1$ \\
\hline Calcium, mg/dL & 11.2 & $8.8-10.6$ \\
\hline Magnesium, mg/dL & 2.5 & $1.8-2.6$ \\
\hline Uricemia, mg/dL & 4.5 & $3.5-7.2$ \\
\hline Proteins, g/dL & 7.6 & $6.6-8.3$ \\
\hline Albumin, g/dL & 4.4 & $3.5-5.2$ \\
\hline Total bilirubin, $\mathrm{mg} / \mathrm{dL}$ & 0.5 & $0.2-1.1$ \\
\hline Direct bilirubin, mg/dL & 0.1 & $0.1-0.4$ \\
\hline Indirect bilirubin, mg/dL & 0.4 & $0.2-0.8$ \\
\hline $\mathrm{LDH}, \mathrm{U} / \mathrm{L}$ & 274 & $0-248$ \\
\hline AST, U/L & 26 & $5-45$ \\
\hline $\mathrm{ALT}, \mathrm{U} / \mathrm{L}$ & 36 & $5-55$ \\
\hline GGT, U/ L & 30 & $10-50$ \\
\hline Cholinesterase, U/L & 5439 & $4500-11400$ \\
\hline$A L P, U / L$ & 118 & $30-120$ \\
\hline Sideremia, $\mu \mathrm{g} / \mathrm{dL}$ & 67 & $70-180$ \\
\hline
\end{tabular}


while he did not complain of visual field deficits. The patient reported persistence of polyuria and polydipsia (about $8 \mathrm{~L} /$ day), which did not change following the introduction of corticosteroid therapy. Blood chemistry tests showed glycemic decompensation (glucose: $320 \mathrm{mg} /$ dL) with plasma hyperosmolality (303 $\mathrm{mOsm} / \mathrm{kg}$ ) and urine hyposmolarity $(158 \mathrm{mmol} / \mathrm{L})$.

\section{Investigation}

Pituitary hormonal function was assessed (Table 3) and frank hypogonadotropic hypogonadism and mild hyperprolactinemia were confirmed. The low TSH value was compatible with concomitant corticosteroid therapy (6). To complete the study of glycemic decompensation, HbA1c (7.7\%), C-peptide $(0.73 \mathrm{ng} / \mathrm{mL}$, reference value: $0.5-2$ ) and antibodies against glutamic acid decarboxylase $(<5 \mathrm{IU} / \mathrm{mL})$ and anti IA2 $(<10 \mathrm{IU} / \mathrm{mL})$ were assessed. The autoimmune origin was therefore excluded and diagnosis of metasteroid diabetes mellitus was made. It was treated with insulin therapy. During the hospitalization, methylprednisolone dose was tapered off, leading to the resolution of mycosis (which was also due to antifungal therapy) and the restoration of glycemic compensation with progressive reduction in the dose of insulin, until full discontinuation. Once normalization of blood glucose levels was obtained, the patient reported improvement in symptoms of polyuria and polydipsia, which however persisted albeit to a lesser extent (water balance: IN $4.5 \mathrm{~L}$ - OUT $5.5 \mathrm{~L}$ ). The patient was affected by the polyuria-polydipsia syndrome (water intake $>3 \mathrm{~L} /$ day and diuresis $>40-50$ $\mathrm{mL} / \mathrm{kg} /$ day), and it was, therefore, necessary to define the etiology. Because of the technical limitations of the $\mathrm{ADH}$ assay (7), and the impossibility of copeptin dosage

Table 3 Laboratory values during treatment with corticosteroid, after nivolumab discontinuation.

\begin{tabular}{|c|c|c|}
\hline Hormone/antibody & Result & Reference values \\
\hline $\mathrm{TSH}, \mu \mathrm{UI} / \mathrm{mL}$ & 0.147 & $0.89-1.76$ \\
\hline FT4, ng/dL & 1.22 & $0.55-4.78$ \\
\hline $\mathrm{TGAb}, \mathrm{U} / \mathrm{mL}$ & 17.10 & $<60$ \\
\hline TPOAb, U/mL & 38.30 & $<60$ \\
\hline Testosterone, ng/dL & $<20.0$ & $181-758$ \\
\hline $\mathrm{LH}, \mathrm{mUl} / \mathrm{mL}$ & 0.4 & $0.8-7.6$ \\
\hline $\mathrm{FSH}, \mathrm{mUl} / \mathrm{mL}$ & 0.6 & $0.7-11.1$ \\
\hline Prolactin, ng/mL & 18.6 & $2.5-17.0$ \\
\hline $\mathrm{GH}, \mathrm{ng} / \mathrm{mL}$ & 0.4 & $<10$ \\
\hline $\mathrm{IGF}-1, \mathrm{ng} / \mathrm{mL}$ & 109 & $43-220$ \\
\hline $\mathrm{ACTH}, \mathrm{pg} / \mathrm{mL}$ & 6 & $0-46$ \\
\hline Cortisol, $\mu g / d L$ & 20.3 & $4.3-22.4$ \\
\hline
\end{tabular}

in our clinical laboratory, we assessed urine osmolarity and plasma osmolality. After just $2 \mathrm{~h}$ of fluid restriction, the onset of hypernatremia contraindicated the water deprivation test's prosecution. Psychogenic polyuria was ruled out due to the presence of low urine osmolarity in association with high plasma osmolality. Afterwards, we decided to evaluate the response to the administration of desmopressin $2 \mu$ g s.c. In Table 4 you can see the values of natremia, plasma osmolality and urine osmolarity before and after the administration of the drug. An increase of $146 \%$ in urine osmolarity after desmopressin allowed us to rule out nephrogenic diabetes insipidus and has made diagnosing central diabetes insipidus possible (8). Antipituitary antibody and anti-rabphilin 3A antibody were not available in our laboratory, therefore they haven't been investigated.

At this point, pituitary MRI scan with gadolinium was performed. The radiological report showed a pituitary stalk substantially in axis, with marked and widespread thickening, up to about $6 \mathrm{~mm}$, associated hyperintensity and contrast enhancement. Neurohypophysis hyperintensity was not displayed. A roundish formation of about $7 \mathrm{~mm}$ in diameter, substantially isointense with respect to the remaining glandular parenchyma in pre-contrast T1- and T2-weighted images and tenuously hypointense in post-contrast image, was observed at the posterior adenohypophyseal portion, in the medianparamedian area. The lesion seemed to cause focal interruption of the sellar floor (Fig. 1). In addition to the MRI examination of the pituitary, post-contrast axial DWI and 3D-T1-FFE sequences were acquired on the entire brain with documented presence of multiple contrast medium enhancement lesions located in the supra and subtentorial regions and in both cerebellar hemispheres (Fig. 2). The neuroradiological data were therefore compatible both with clinical suspicion of metastatic localization and with the hypothesis of hypophysitis secondary to therapy with nivolumab.

\section{Treatment}

The patient underwent replacement therapy with DDVAP sublingual tablets $60 \mu \mathrm{g}$ in divided dose twice daily, with normalization of the water balance ( $2 \mathrm{~L}$ in balance).

Nine days after hospitalization, the patient was discharged with the following diagnosis 'Partial hypopituitarism (diabetes insipidus and hypogonadism) secondary to probable nivolumab-induced infundibuloneurohypophysitis and anterohypophyseal metastasis, in a patient suffering from metastatic hypopharyngeal 
Table 4 Plasma osmolality and urine osmolarity before and after desmopressin administration.

\begin{tabular}{lcc}
\hline Sampling time & & Natremia (mEq/L) \\
\cline { 1 - 1 } Before desmopressin & 146 \\
7:00 h & & 142 \\
Desmopressin $2 \mu g$ s.c. injection: $10: 00 \mathrm{~h}$ \\
12:30 h & - \\
15:30 h &
\end{tabular}

carcinoma; multiple cerebral and cerebellar new on-set metastases and metasteroid diabetes mellitus'. The patient was started on replacement therapy with DDVAP sublingual tablets $60 \mu \mathrm{g}$ in divided dose twice daily, $2 \%$ testosterone gel 4 puffs/day and methylprednisolone dose was tapered off until $4 \mathrm{mg}$, pending execution of ACTH test to evaluate the possible suppression of the hypothalamic-pituitary-adrenal axis. As the pituitary impairment was well compensated by the replacement therapy, no contraindication was given to the resumption of nivolumab therapy with necessary monitoring of the residual pituitary function.

\section{Outcome and follow-up}

The patient was re-evaluated by the oncologist and underwent total body CT scan for disease re- staging. In addition to the already known secondary malignancy in the lung, new-onset adrenal, pancreatic, skeletal metastases and peritoneal carcinosis were found.

Due to poor response to nivolumab therapy and rapid disease progression, treatment was stopped permanently. The patient died about 50 days after hospital discharge.

\section{Discussion}

The hypophysitis is an adverse event more characteristic of anti-CTLA4, and only to a lesser extent than anti-PD1. Patients with immune therapy-induced hypophysitis mostly present with multiple hormonal deficits, mainly of thyrotropin (84\%), corticotropin $(80 \%)$ and gonadotropins (76\%); the involvement of the posterior pituitary is exceptional (2). So far, there are only two case reports described in the literature about anti PDL1 (3) and anti PD1- related central diabetes insipidus (4). The average time to onset of endocrinological side effects is 9 weeks from the start of treatment (with a range from 5 to 36 weeks). Considering the high incidence of these adverse events following therapy with immune-checkpoint, it is essential to research any clinical signs suggestive of iRAes before each cycle of therapy; when a suggestive symtom is found, it is essential to evaluate hormonal tests to identify endocrinopathy (9). In general, the oncological clinical practice involves the administration of high-dose corticosteroids for most iRAes. As regards specifically the pituitary, a recent consensus has highlighted how the use of high-dose corticosteroids is to be reserved exclusively for cases of adrenal crisis, severe headache and visual field deficit (10). In fact, corticosteroid therapy would be able to resolve the signs of local inflammation, but not the hormonal deficit. In addition, high-dose therapy would be responsible for major complications, some serious enough to require hospitalization (11). To confirm this, our patient had not received any benefit from corticosteroid therapy, but his general health condition worsened as a result of diabetes mellitus, dehydration and fungal infection onset. Besides the patient suffered from impaired fasting glucose, and glucocorticoid treatment precipitated a prediabetes condition. The etiology of diabetes mellitus in our patient under both corticosteroids and immune-
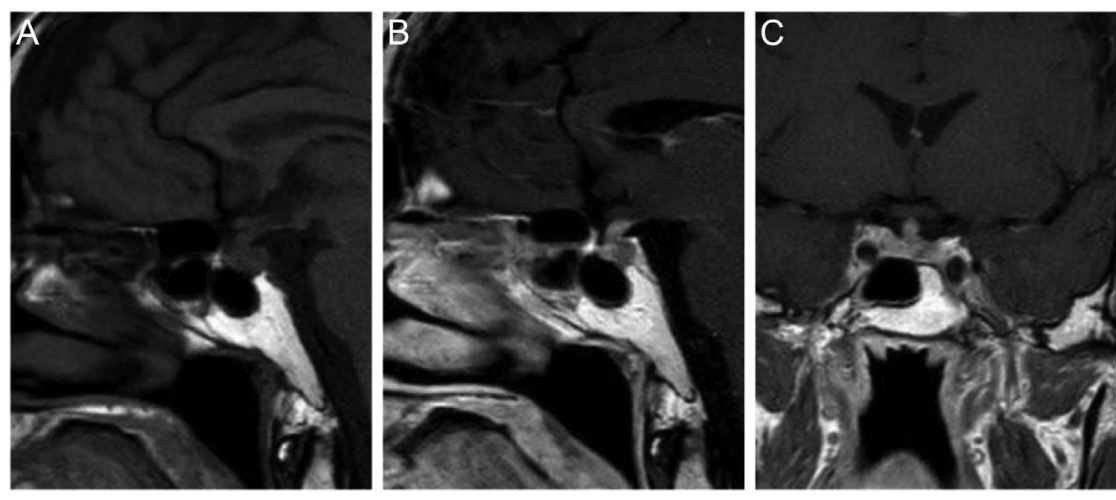

Figure 1

Pre-contrast (A) and postcontrast T1-weighted sagittal (B) and coronal (C) MR image demonstrates a diffuse enlargement of the pituitary stalk. The pituitary gland shows heterogenous enhancement due to a hypotense lesion, causing focal destruction of the sellar floor. 

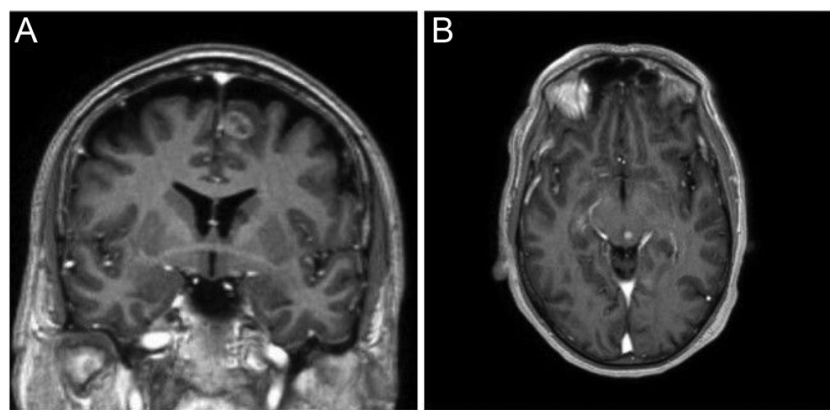

Figure 2

Post-contrast T1-weighted MR image of the brain demonstrates multiple metastatic lesions at the grey-white matter junction in the frontal lobe $(A)$ and in the midbrain, close to the Sylvian aqueduct (B).

checkpoint inhibitors treatment, was, at first, uncertain. In fact, among the autoimmune adverse events typical of immune-checkpoints inhibitors, there is also diabetes mellitus (12). The autoimmune form of origin was however excluded due to the absence of autoimmunity, the pancreatic reserve and the normalization of blood glucose levels following the steroid tapering.

The persistence of polyuria and polydipsia led us to investigate the origin of the polyuria-polydipsia syndrome, confirming the diagnosis of diabetes insipidus following the evaluation of plasma osmolality and urine osmolarity before and after DDVAP.

The diabetes insipidus in our patient could have been mainly due to two causes: the presence of metastases in the pituitary region or the onset of hypophysitis secondary to nivolumab therapy.

A recent observational retrospective study assessed the clinical, biochemical and radiological differences in patients with neoplastic and non-neoplastic pituitary stalk pathology. Statistically significant differences were found for the greater preponderance of diabetes insipidus and hypogonadism in subjects suffering from neoplastic pathology compared to those suffering from nonneoplastic pathology; with regard to radiological images, the features that have reached statistical significance were the thickness of the stalk and the heterogeneity of the contrast enhancement after administration of contrast agent. Specifically, all thickenings greater than $9.1 \mathrm{~mm}$ in diameter in the post-contrast coronal section in the upper part of the stalk and greater than 5.6 in the coronal section in the lower part were confirmed as neoplastic with a sensitivity of $100 \%$ (13). Our patient had biochemical and radiological characteristics consistent with the coexistence of neurohypophysitis (stalk thickness $6 \mathrm{~mm}$, hyperintensity under the Gadolinium enhancement, and no evidence of neurohypophysis hyperintensity) and pituitary metastasis (roundish formation, at the posterior adenohypophyseal portion, causing focal interruption of the sellar floor). To define the exact etiology of the pituitary pathology, histological confirmation through biopsy sampling would have been necessary; however, because of the patient's critical health condition, it was not possible to perform. In the absence of histological confirmation, we believe it is likely that both could have played a role in the onset of diabetes insipidus secondary to the pituitary stalk interruption syndrome, as a result of metastatic compression and drug therapy-induced hypophysitis.

\section{Declaration of interest}

The authors declare that there is no conflict of interest that could be perceived as prejudicing the impartiality of the research reported.

\section{Funding}

This research did not receive any specific grant from any funding agency in the public, commercial or not-for-profit sector.

\section{Patient consent}

Informed consent was obtained from the patient for publication of this report.

\section{Author contribution statement}

All the authors have contributed to the writing and editing of this manuscript and the physician responsible for the patient is one of the authors.

\section{References}

1 Darvin P, Toor SM, Sasidharan Nair V \& Elkord E. Immune checkpoint inhibitors: recent progress and potential biomarkers. Experimental and Molecular Medicine 201850 1-11. (https://doi. org/10.1038/s12276-018-0191-1)

2 Albarel F, Castinetti F \& Brue T. MANAGEMENT OF ENDOCRINE DISEASE: Immune check point inhibitors-induced hypophysitis. European Journal of Endocrinology 2019181 R107-R118. (https://doi. org/10.1530/EJE-19-0169)

3 Zhao C, Tella SH, Del Rivero J, Kommalapati A, Ebenuwa I, Gulley J, Strauss J \& Brownell I. Anti-PD-L1 treatment induced central diabetes insipidus. Journal of Clinical Endocrinology and Metabolism 2018103 365-369. (https://doi.org/10.1210/jc.2017-01905)

4 Deligiorgi MV, Siasos G, Vergadis C \& Trafalis DT. Central diabetes insipidus related to anti-programmed cell-death 1 protein active immunotherapy. International Immunopharmacology 202083106427. (https://doi.org/10.1016/j.intimp.2020.106427)

5 Shimon I. Metastatic spread to the pituitary. Neuroendocrinology 2020 110 805-808. (https://doi.org/10.1159/000506810) 
6 Burch HB. Drug effects on the thyroid. New England Journal of Medicine 2019381 749-761. (https://doi.org/10.1056/ NEJMra1901214)

7 Christ-Crain M. EJE AWARD 2019: new diagnostic approaches for patients with polyuria polydipsia syndrome. European Journal of Endocrinology 2019181 R11-R21. (https://doi.org/10.1530/EJE-190163)

8 Miller M, Dalakos T, Moses AM, Fellerman H \& Streeten DH. Recognition of partial defects in antidiuretic hormone secretion. Annals of Internal Medicine 197073 721-729. (https://doi. org/10.7326/0003-4819-73-5-721)

9 Joshi MN, Whitelaw BC, Palomar MT, Wu Y \& Carroll PV. Immune checkpoint inhibitor-related hypophysitis and endocrine dysfunction: clinical review. Clinical Endocrinology 201685 331-339. (https://doi.org/10.1111/cen.13063)

10 Puzanov I, Diab A, Abdallah K, Bingham CO 3rd, Brogdon C, Dadu R, Hamad L, Kim S, Lacouture ME, LeBoeuf NR, et al. Managing toxicities associated with immune checkpoint inhibitors: consensus recommendations from the Society for Immunotherapy of Cancer (SITC) Toxicity Management Working Group. Journal for Immunotherapy of Cancer 20175 95. (https://doi.org/10.1186/s40425017-0300-z)

11 Lammert A, Schneider HJ, Bergmann T, Benck U, Krämer BK, Gärtner R, Metzner C, Schöfl C \& Berking C. Hypophysitis caused by ipilimumab in cancer patients: hormone replacement or immunosuppressive therapy. Experimental and Clinical Endocrinology and Diabetes 2013121 581-587. (https://doi. org/10.1055/s-0033-1355337)

12 Stamatouli AM, Quandt Z, Perdigoto AL, Clark PL, Kluger H, Weiss SA, Gettinger S, Sznol M, Young A, Rushakoff R, et al. Collateral damage: insulin-dependent diabetes induced with checkpoint inhibitors. Diabetes 201867 1471-1480. (https://doi.org/10.2337/ dbi18-0002)

13 Sbardella E, Joseph RN, Jafar-Mohammadi B, Isidori AM, Cudlip S \& Grossman AB. Pituitary stalk thickening: the role of an innovative MRI imaging analysis which may assist in determining clinical management. European Journal of Endocrinology 2016175 255-263. (https://doi.org/10.1530/EJE-16-0455)

Received in final form 11 November 2020

Accepted 7 January 2021 